\title{
Acne - therapeutic challenges to the cooperation between a dermatologist and a cosmetologist
}

\author{
Zygmunt Adamski ${ }^{1}$, Justyna Gornowicz-Porowska², Daria Sobkowska², Klaudyna Kaszuba³ ${ }^{3}$ Rafał Czajkowski ${ }^{4}$ \\ ${ }^{1}$ Chair and Clinic of Dermatology, Poznan University of Medical Sciences, Poznan, Poland \\ ${ }^{2}$ Clinic of Natural Medicinal and Cosmetic Raw Materials, The Laboratory of Practical Cosmetology, Poznan University \\ of Medical Sciences, Poznan, Poland \\ 'Specialist Doctors' Consulting Rooms DERMED, Lodz, Poland \\ ${ }^{4}$ Chair of Dermatology, Department of Dermatology and Venerology, Faculty of Medicine, Ludwik Rydygier Medical College \\ in Bydgoszcz, Nicolaus Copernicus University in Torun, Poland
}

Adv Dermatol Allergol 2021; XXXVIII (1): 21-31

DOI: https://doi.org/10.5114/ada.2021.104273

\begin{abstract}
Acne vulgaris is one of the most common skin diseases in adolescence, causing a significant negative impact on the quality of life of young people who - in a large percentage of cases - do not accept their appearance. The complex pathogenesis of this disease requires appropriate preventive and curative treatment from both a dermatologist and a cosmetologist. The aim of the study was to discuss the methods currently used by dermatologists in local therapy for mild and intermediate forms of acne vulgaris, and to provide an overview of possible preventive and caring activities in the cosmetologist's office. Possible directions of cooperation between the dermatologist and the cosmetologist in the prevention of acne relapse and treatment of possible acne complications were also discussed.
\end{abstract}

Key words: acne vulgaris, local therapy, cosmetology procedures.

\section{Introduction}

Acne vulgaris is the most common skin disease associated with a dysfunction of sebaceous glands. It is characterised by the presence of retentional lesions on the skin, in the so-called primary seborrhoea areas, in the form of whiteheads and blackheads. Subsequently different inflammatory lesions can be observed in the form of papules, pustules, cysts which may heal with leaving disfiguring scars. A significant part of youth, aged 1318 years, struggles with acne. However, increasingly, acne tends to occur in mature age (22-30 years). The disease poses a significant medical problem affecting not only the physical sphere, but it also considerably affects the mental condition of the ill person $[1,2]$.

The occurrence and course of the acne is influenced by many factors. The main ones include: excessive keratosis of the sebaceous gland outlets, increased sebum production during pubescence, and the presence of anaerobic bacteria Cutibacterium acnes in the area of sebaceous glands, and the development of the inflammatory condition of different intensity. Besides that, there are also considered genetic factors, immunological factors, hormonal conditions, stress-inducing conditions, and bad eating habits [3-5].

The environmental conditions significantly affecting the development and course of acne include the following:

- professional, cosmetic factors [5-7],

- medicinal factors - anti-depressants, anabolic steroid hormones, glucocorticosteroids, ACTH, anti-epileptics, anti-tubercular medicines, halides, vitamin $\mathrm{B}_{12}$, barbiturates [8-11],

- chlorine and halogen derivatives [12],

- psychological factors (stress) [13],

- dietary factors (hyperglycaemic food, milk and insulinotropic dairy products) [14-16],

- smoking $[17,18]$.

The recognition of new mechanisms of the disease pathogenesis and development of new therapy methods offered an efficient help to persons suffering from even the most severe forms of acne. At present, in cases difficult to treat, it is increasingly recommended to apply local preparations (retinoids, benzoyl peroxide, antibiot-

Address for correspondence: Zygmunt Adamski MD, PhD, Chair and Clinic of Dermatology, Poznan University of Medical Sciences, Poznan, Poland, e-mail: zadamski@ump.edu.pl Received: 28.03.2020, accepted: 21.07.2020. 
ics) and take oral medicines (antibiotics, retinoids, hormones) $[5,19,20]$.

To prevent development of severe inflammatory forms of acne which, even after an intensive treatment leave scars (atrophic, hypertrophied scars) or keloids, it is desired and appropriate to take preventive and treatment actions at very early stages of the disease. An experienced dermatologist or cosmetologist should be able to recognise and foresee further development of the disease and introduce appropriate actions.

An important role of cosmetologists to whom patients come in the first place, is widely understood prevention through actions based on individually adjusted care treatments, proper education of the patient/client as regards proper care for the skin and its cleansing. Depending on the severity of seborrhoeic manifestations on the facial skin and the areas rich in sebaceous glands, the appropriate treatment methods are chosen. Inflammatory disease effects are treated by a dermatologist with an active support of a cosmetologist to keep the skin free from acne changes for a long time. Cooperation between a dermatologist and a cosmetologist can improve the long-term therapeutic effect, and in the consequence, also affect the satisfaction of the patient/client [21].

\section{Aim}

The aim of the study was to discuss the methods currently used by dermatologists in local therapy of acne vulgaris, and to provide an overview of possible preventive and caring activities in the cosmetologist's office.

\section{Therapeutic proceedings in the dermatologist's office}

Acne vulgaris is a disease of multifactorial, complex etiopathogenesis in the course of which a complex interaction between endogenous and exogenous factors and their effect on pilosebaceous units [20] takes place. Changes in the sebum composition and increased sensitivity to androgens stimulate the inflammatory process in which $C$. acnes and congenital immunity play the main role in the evolution of the pilosebaceous unit outlets keratosis and propagation of the inflammatory condition $[5,9,20]$. Development of the severe forms of acne depends on the degree of intensification of this condition.

The main purpose of dermatological treatment, according to the Consensus of the Polish Dermatological Society is the liquidation or alleviation of the inflammatory manifestations and scar formation prevention. The proceedings should mainly be based on good communication with the patient, resulting in the observance of the recommended treatment methods, and explanation of the course of treatment and the need to apply maintenance treatment. It should be noted that local therapy is the basic treatment of both non-inflammatory and in- flammatory lesions, and it is sufficient in about $60 \%$ of patients [5, 19, 20, 22].

\section{Local therapy in acne vulgaris}

In local therapy of acne, the following need to be considered:

- monotherapy,

- combined therapy,

- treatment supplementary to the general dermatological treatment,

- maintenance treatment,

- physical treatment.

Local preparations should be applied to the entire area of the skin with lesions, as in those areas microcomedones occur which are often difficult to see with bare eye and are the basis for development of comedones and most inflammatory acneiform lesions [19, 22].

Local therapy should be focused on the liquidation of basic pathogenetic phenomena such as:

- excessive keratosis of pilosebaceous unit outlets (keratolytic and anti-comedones activity),

- growth of $C$. acnes (anti-inflammatory and antibacterial activity),

- excessive seborrhoea (anti-seborrhoea, anti-androgynous activity).

Local therapy with the use of a monotherapy or combined therapy is applied in treatment of mild and moderate forms of acne. It may also supplement the general treatment and constitute a maintenance therapy after the regression of symptoms in general treatment [23-26].

In monotherapy of such forms as comedonal acne and comedo-papular acne, retinoids, benzoyl peroxide and azelaic acid can be applied [26, 27].

Antibiotics should not be used in monotherapy as this may induce drug resistance, which has been increasingly observed during the recent years $[26,27]$.

\section{Local retinoids}

Five types of local retinoids are used in acne treatment. These include first-generation retinoids:

- tretinoin (all-trans-retinoic acid),

- isotretinoin (13-cis-retinoic acid) and

- retinaldehyde.

Third-generation retinoids include: adapalene and tazarotene, which are characterised by more selective affinity to retinoid receptors.

Locally administered retinoids:

- demonstrate an inhibitory effect on sebaceous glands - anti-seborrhoea activity,

- normalise keratosis of pilosebaceous unit outlets keratolytic activity, 
- release corneous masses from follicles outlets and facilitate sebum evacuation from the glands - keratolytic activity,

- prevent the formation of micro-comedones - anticomedogenic activity,

- inhibit the development of C. acnes,

- indirectly reduce the intensity of the inflammatory reaction through a change of the micro-environment unfavourable for the development of $C$. acnes.

Regardless of the above indirect effect, tretinoin, tazarotene, and adapalene demonstrate direct antiinflammatory properties, while adapalene seems to be the most effective of them [28-30].

Anti-inflammatory effect is induced by the suppression of TLR expression, inhibition of the production of some interleukins and modulation of T CD4+, CD8+ lymphocytes and polymorphonuclear leukocytes (PMN).

Locally applied retinoids may intensify the penetration of sebaceous glands by other medicines applied in acne therapy (e.g. benzoyl peroxide or locally administered antibiotics) and bring a positive effect of the combined activity [31, 32].

Retinoids are characterised by various strength of action, but similar adverse effects. These include:

- skin inflammation of the type ro-dermatitis, demonstrated by erythema, exfoliation, dryness, burning sensation, itching (particularly intensified in persons with a history of atopy),

- hypersensitivity to solar radiation,

- hypo- and hyperpigmentation, ectropion and lesions of the contact dermatitis type [31-33].

Due to the side effects, it is recommended that those medicines are applied once a day, in the evening, on the entire area of the skin with acneiform lesions. Moreover, it is recommended that the preparation is applied 1520 min after gentle cleansing of the skin covered with acneiform lesions. Treatment with local retinoids, especially in patients with dry skin (a large number of whiteheads) and predisposition to irritations, should begin with the following scheme:

- $1 \times$ in the evening, every 3 days (week 1 ),

- $1 \times$ in the evening, every 2 days (week 2 ),

- $1 \times$ in the evening, every day (week 3, 4 and on).

In the therapy with local retinoids it is recommended:

- to use non-comedogenic moisturisers (alleviating irritations),

- to avoid simultaneous application of irritant preparations,

- to protect eyes, mouth, and mucous membranes,

- to apply photoprotection,

- to make the patient aware that acneiform lesions may intensify in the first 2-4 weeks of the treatment.

At the end of the $2^{\text {nd }}$ month of therapy, considerable clinical improvement is observed and the irritation disappears. Therefore, patients must be made aware of that so as to patiently adhere to the recommended therapy [23].
An important issue associated with the use of local retinoids is contraception in women of reproductive age. Tretinoin and adapalene, due to more lipophilic nature and low systemic absorption, belong to category C, according to the American Food and Drug Administration (FDA), which means that medicines from this group can be applied during pregnancy only if the doctor believes that the expected benefit for the mother outweighs the potential risk for the foetus. Whereas, isotretinoin and tazarotene belong to category $X$ according to FDA, which means that these medicines are strictly forbidden to be used during pregnancy.

\section{Benzoyl peroxide}

Benzoyl peroxide (BPO) is a unique medicine used in local therapy of acne. Its mechanism of action includes:

- antibacterial activity on C. acnes - it reduces the number of bacteria by ca. $90 \%$,

- despite long-lasting therapy, bacteria remain sensitive to BPO (drug resistance is not developed),

- wide spectrum of antibacterial activity (Cutibacterium acnes, Staphylococcus sp.),

- demonstrates slightly less keratolytic activity in comparison to retinoids, and in the consequence less comedolytic effect also on micro-comedones.

This makes the medicine very useful; it may be applied both in monotherapy and in combined therapy of mild and moderate forms of acne vulgaris [34-36].

However, the medicine has also some flaws which must be specifically communicated to the patient under treatment:

- it shows skin irritant properties, therefore local therapy should be started with the lowest concentrations and less frequent applications (it is available in concentrations from $4 \%$ to $10 \%$ in creams and solutions),

- may cause discolouration of clothes.

However, as regards local therapy of acne, it is a basic medicinal product.

\section{Azelaic acid}

It is intended to focus on the most important elements of acne pathogenesis and consists in:

- antibacterial activity (both toward C. acnes, and S. epidermidis),

- anti-inflammatory activity through the inhibition of polymorphonuclear neutrophils,

- poor keratolytic activity,

- poor anti-seborrhoea activity through the inhibition of enzyme 5a-reductase,

- inhibition of melanogenesis and reduction of postinflammatory discolourations in acne,

- causing no resistance to antibiotics.

This medicine is suitable for use in monotherapy. It is characterised by weaker activity as compared with local retinoids but it is tolerated better $[37,38]$. 
It is used twice a day in the form of creams and gels in the concentration of $15-20 \%$, and as one of the ingredients in peelings.

\section{Local antibiotics}

The most recommended and frequently used antibiotics in Poland in local therapy of acne are:

- clindamycin in the form of 1\% fluid, emulsion and gel,

- erythromycin in 2-4\% solution,

- davercin - erythromycin cyclocarbonate in the form of gel (2.5\%), fluid and ointment (1\%).

Erythromycin cyclocarbonate acts 2-3 times stronger than erythromycin, has 3 times longer half-life, lower minimal inhibitory concentration (MIC), and shows higher durability in acidic environment $[39,40]$.

In the recent years there has been observed a common increase of resistance to antibiotics for external use, especially erythromycin and clindamycin. In European countries, the resistance of $C$. acnes to antibiotics reaches from $51 \%$ in Hungary to $94 \%$ in Spain [41].

This may result from the fact that medicine resistance of $C$. acnes is distributed through contact (e.g. between siblings, patient and doctor) [42]. Quite often there is a cross-resistance between erythromycin and clindamycin, and drug resistance can be transmitted to other pathogens of the skin, especially S. haemolyticus and S. epidermidis.

The mechanism of antibiotics action in acne consists in:

- limitation of the local inflammatory condition (inhibition of neutrophils chemotaxis, cytokines production, and macrophages function),

- reduction of $C$. acnes count in sebaceous glands,

- reduced activity of bacterial lipases, and in the consequence the change of free fatty acids (FFA) to triglycerides ratio, in favour of the esters,

- quick disappearance of papules and pustules, especially in the comedo-papular-pustular acne,

- locally applied antibiotics are well-tolerated, side effects occur seldom (concern 0-5\% of patients), and the intensity of the disease is mild (reddening, itching, burning sensation, skin dryness),

- both clindamycin and erythromycin can be used during pregnancy (category B according to FDA) [43].

The main principles of local antibiotics application.

Antibiotics should be used only when needed.

The patient must be educated about the regularity of medicine intake, the prohibition to discontinue the therapy, or to extend the therapy without need.

We discontinue the use of antibiotics when the inflamed lesions vanish, and they should not be used for more than up to 12 weeks $[37,38]$.

If the lesions do not vanish, the external medicine must be changed or general antibiotic must be used.
Benzoyl peroxide (BPO) should be used for at least 5-7 days between the periods of antibiotic therapy, or simultaneously with the antibiotic $[37,43]$.

Simultaneous application of local antibiotics and general antibiotics without BPO must be avoided as this does not ensure synergistic activity, and only increases the probability of developing resistance to antibiotics.

Comment: According to the Polish Dermatological Society, monotherapy with local antibiotics should not be used either in the phase of active treatment of acneiform lesions or during maintenance therapy.

Preparations containing antibiotics are generally welltolerated and readily used by patients. Patients with acne are often treated with many antibiotics, and the microbiota of their skin is exposed to the development of drug resistance. Therefore, the use of local antibiotics should be limited to the necessary clinical indications.

\section{Local combined therapy}

Acne vulgaris is a disease of a very complex pathogenesis which often requires the application of preparations with different mechanisms of action. Combined therapy may be adjusted individually to each clinical form of acne. It is used to increase the effectiveness of the therapy, shorten the period of active treatment and minimize potential adverse effects.

Three most frequent forms of combined therapy include:

- alternating therapy with medicines of different mechanisms of action (antibacterial and anti-inflammatory/ keratolytic and comedolytic),

- application of various preparations at different times of a day (e.g. for day - antibiotic/ for night - benzoyl peroxide)

- combined preparations "two-in-one" (e.g. antibiotic with benzoyl peroxide, or antibiotic with local retinoid).

Alternating therapy is recommended for long-term therapy with antibiotics, which prevents $C$. acnes drug resistance that reduces the effectiveness, extends the therapy and causes more frequent disease relapse [35, 37, 44].

As the basic proceeding strategy in local therapy which is to reduce the development of drug resistance, it is recommended to apply simultaneously (at different times of a day, or simultaneously in case of combined preparations) with benzoyl peroxide or retinoid [37, 45].

Introduction of combined preparations results not only from the synergistic activity of respective ingredients, but also from the possibility to improve the doctorpatient cooperation line. At present it is believed that proper use of the recommended therapy by the patient is the basis for successful treatment effects, especially in the youngest patients aged 13-17 years, who form the largest group of patients [37, 42]. 
The most frequently combined local anti-acne medicines in Poland are:

- erythromycin $4 \%+$ zinc acetate $1.2 \%$,

- tretinoin $0.025 \%$ + erythromycin $4 \%$,

- isotretinoin $0.05 \%$ + erythromycin $2 \%$,

- benzoyl peroxide $5 \%$ + clindamycin $1 \%$,

- benzoyl peroxide $2.5 \%$ + adapalene $0.1 \%$,

- clindamycin 1\% + tretinoin $0.025 \%$.

When assessing the benefits of the application of preparations combining retinoids and antibiotics, it is emphasised the increased effectiveness (keratolytic medicine + antibacterial medicine) increase penetration by the antibiotic and reduces irritant activity. The combinations containing BPO (5\%) and clindamycin or erythromycin, are characterised by considerable effectiveness and very good tolerance [46]. The research by Burkhart et al. [47] have proven that the combination of $\mathrm{BPO}$ and the antibiotic may result in better antibacterial effectiveness as compared to the respective compounds used separately.

Adapalene + BPO is another complex preparation of significantly increased activity reducing the inflamed and non-inflamed lesions. It has been proven that the preparation is characterised by the increased effectiveness in acne therapy in comparison to respective compounds, and significantly reduces the population of $C$. acnes [29].

At present, the most reasonable treatment seems to be the therapy with compound preparations containing the combination of BPO and the antibiotic or retinoid. The effectiveness of those therapies is very similar although the preparations without the retinoid demonstrate a slightly quicker action and less intense adverse effects, especially in the initial period of the therapy.

\section{Local preparations in maintenance treatment}

The benefits of local maintenance treatment after the end of the therapy of active acneiform lesions are indisputable. In acne maintenance therapy the following are applied:

- retinoids preventing formation of micro-comedones (tretinoin, tazarotene) [37, 48],

- new generation retinoids with reduced irritant properties - adapalene [27, 37],

- monotherapy with azelaic acid which acts on all elements of acne pathogenesis [27].

\section{Therapeutic proceedings in the cosmetologist's and/or dermatologist's office}

Obviously, a person performing the cosmetic procedure must have adequate skills. In light of this, for the treatment of any kind of medical skin condition, a dermatologist should always be the first choice.

\section{Skincare proceedings}

Proper skincare proceedings in case of acneiform complexion is of particular significance at the early stages of acne. It may contribute to the improvement of the patient's complexion and constitutes a very important, first element of their proper health education. It must be remembered that improper care for such complexion may intensify acne manifestations.

Proper care for acneiform skin:

- plays a very important role supporting the treatment,

- in mild forms of the disease it may cause the vanishing of lesions.

Facial skin (complexion) in acne usually demonstrates the features of seborrhoea or mixed complexion. In the presence of a large number of retentional lesions (open and closed comedones), it shows the features of dry complexion prone to develop irritation with the manifestations of superficial peeling, especially in the area of cheeks and lateral parts of the face where the number of sebaceous glands is much smaller. Seborrhoeic areas, i.e. the places with the largest number of sebaceous glands in humans, are:

- forehead, central part of the face (nose and the surrounding area) i.e. the so called T-zone,

- shoulders and the area between the shoulder blades,

- upper, anterior part of the chest with the sternum area.

In case of mixed complexion and in persons with the coexisting atopy (skin dryness due to genetic filaggrin deficiency), the adjustment/choice of proper skincare products is particularly difficult. They should contain linoleic or g-linolenic acid or the constituents of the natural moisturising factor (NMF) - urea and pyrrolidone carboxylic acid [49].

Like the medical treatment, skincare should focus on four basic pathogenetic mechanisms of the disease (excessive seborrhoea, keratosis of pilosebaceous unit outlets, bacterial factor, inflammatory condition) [49]. Dermocosmetics for acneiform complexion should contain properly adjusted active components displaying the following activities:

- anti-seborrhoea,

- peeling and promoting the process of cell regeneration,

- moisturising and mitigating the skin,

- characteristic for mattifying preparations, ensuring fresh looks and the feeling of comfort.

The rules of proper care for acneiform complexion:

- Acne skin is easily prone to irritation:

- Too vigorous washing and scrubbing may cause the intensification of the inflammatory phase of acne.

- Too strong drying effect promotes the inflammatory condition, increases sebum secretion and keratosis of pilosebaceous unit outlets.

- Coarse flannels cause the tearing of hair follicles, thus increasing the number of inflammatory lesions. 
- Skin cleansings in acne should remove sebum excess and exfoliated epidermis, reduce secretion of sweat glands with the use of gentle preparations in the form of micellar fluids, gel preparations and soaps with acidic $\mathrm{pH}$ (5.0-7.0).

- One should not use basic soaps and alcohol containing tonics which are irritant to skin.

- The so-called antibacterial soaps most frequently contain chlorhexidine or triclosan, and they do not affect the bacteria present in acne.

- While choosing skincare creams and preparations, patients with acne should pay attention to the cosmetics which do not promote the development of comedones (non-comedogenic).

- Such preparations should not contain comedogenic hydrocarbons such as petrolatum and paraffin.

- Cosmetics for acneiform complexion should be based on gels, emulsions, containing in their substrates silicone derivatives (cyclomethicone, dimethicone), and not on oils.

- Beneficial cosmetics are face washing fluids containing benzoyl peroxide which demonstrate strong antibacterial activity, also on pathogenic flora of the skin.

\section{Anti-comedones and anti-seborrhoea activity}

The activities aiming at the reduction of the sebum secretion are the main objectives of the cosmetologist's and dermatologist's actions as the excessive production of sebum and any changes in its composition belong to the most important pathogenetic factors of acne.

Anti-seborrhoea products include:

- Creams, emulsions and solutions with $\alpha$-hydroxy acids (AHA) or $\beta$-hydroxy acids (BHA) in their composition, which first of all display keratolytic (peeling) activity and control sebum secretion and to a lower extent also inhibit the secretion,

- skincare preparations containing low concentrations of glycolic acid, salicylic acid, lactic acid, azelaic acid $[28,46]$,

- peelings with the use of salicylic acid, pyruvic acid, glycolic acid, and Jessner's solution (resorcinol, salicylic acid, lactic acid in ethanol).

\section{Face skin matting}

Mattifying preparations, liquidating the shining of face skin (typical for oily skin) are intended to improve the aesthetics of the face and its good cosmetic effect. They are most frequently used as make-up foundation.

In acne, the make-up is used to improve the disadvantageous look of the complexion, however, the selection of cosmetics is of great significance from a practical point of view.

Preparations used for the correction of the existing skin lesions (gels, tonics, loose powders, clays) should not clog the sebaceous gland ducts, which means they should not demonstrate comedogenic effects. Correction make-up is made with the observance of the basic hygiene rules with appropriate disinfection of tools used. During putting the make-up on, one must follow the basic hygiene rules to eliminate the risk of skin infection $[5,49]$.

\section{Cosmetic procedure}

Skincare cosmetology procedures available in cosmetic offices are very diversified. When choosing the right method improving the looks of acneiform skin, one should first of all take into account the type of skin (dry, sensitive, couperose skin etc.), the severity of lesions and the clinical type of acne.

The procedures of mechanical cleansing of acneiform skin from non-inflammatory lesions, and even more importantly, the inflammatory lesions should be totally refrained from as they may cause intensification of the inflamed lesions, scars creation, discolouration, i.e. the complications difficult to treat. Also surgical interventions, in case of large abscesses or cysts should be made carefully by puncturing and not cutting. Proper proceedings in each case of this chronic disease should be specified by a dermatologist [28, 46, 49].

\section{Chemical peelings}

The commonly known and verified methods for persons with acne vulgaris include procedures with the use of chemical peelings. Such procedures should be preceded with appropriate preparation of the skin and should be supplementary to acne therapy (a cooperation between the dermatologist and cosmetologist). It is extremely important that the chemicals used for peeling, and the technique of performing the treatment is properly and individually adjusted. Their main role is to regulate the keratinization process, which is the ability to remove dead stratum corneum cells. Moreover, some chemical peelings may have an inhibitory effect on the processes of skin hyperpigmentation and improve the looks of scars. Depending on the concentration used or the type of exfoliating substance, its ability to permeate the epidermis layers differs, and so does the depth and effectiveness of the peeling. Procedures of this type supplement the treatment of active acneiform lesions, and even more frequently are used to treat permanent post-acneiform lesions.

Deep peelings with stronger concentrations of preparations should be performed by experienced dermatologists and plastic surgeons, and the work with lower concentrations is admissible at the cosmetologist's office. After the treatment one should apply proper care proceedings, and UVA and UVB radiation filters [5, 28, 46, 49]. 


\section{Combined peelings}

The most frequently used acids include azelaic acid, glycolic acid, mandelic acid, or salicylic acid [50-52]. Whereas it is worth mentioning that the combination of some of the above-mentioned compounds may give much better effects on the skin. The research by Choi et al. [53] has proven that application of several substances of exfoliating properties gave better and quicker cosmetic effects on face skin lesions in patients with acne vulgaris, as compared with the application of one kind of peeling. The study covered 45 patients with mild to moderate acne. In the study three subgroups were distinguished. In the first group, modified Jessner's solution with 20\% trichloroacetic acid (TCA) was applied on the right side of the face, and 30\% TCA was applied on the left side of the face. In the second group, the combination of $10 \%$ mandelic acid and $20 \%$ salicylic acid was applied on the right side of the face and 30\% salicylic acid was applied on the left side of the face. Facial skin of the last group was treated with Jessner's solution and TCA the right side, and the left side $-10 \%$ mandelic acid and $20 \%$ salicylic acid. The treatments were performed every 2 weeks, for the period of 3 months in the total number of 6 [54].

\section{Azelaic acid}

Azelaic acid $\mathrm{HOOC}-\left(\mathrm{CH}_{2}\right) 7-\mathrm{COOH}$ is an organic chemical compound of the group of dicarboxylic acids. Due to antibacterial (inhibits the growth of $C$. acnes), anti-inflammatory, keratolytic, anti-seborrhoea activity, inhibiting the synthesis of melanin (lightening the discolourations), azelaic acid is used in inflammatory forms of acne vulgaris and rosacea, and in the treatment of discolouration. In one publication concerning the use of $30 \%$ azelaic acid in a group of 35 women with acneiform lesions on the face skin, its beneficial effect on the improvement of skin condition was proven. The therapy lasted for 3 months -6 meetings every 2 weeks. The result was a decreased number of acneiform lesions on the skin and reduction of seborrhoea [55]. It is presumed that the application of azelaic acid in combination with clindamycin, benzoyl peroxide or another $\alpha$-hydroxy acid may give a better therapeutic result [55].

Very frequent adverse effects include: burning sensation, erythema, and itching. Frequent adverse effects are: exfoliation of the epidermis, dryness, discolouration, irritation in the place of administration. Rare adverse effects are: intensification of seborrhoeic dermatitis, hypersensitivity with skin swelling, erosions. It is applied locally, in patients aged $12+$, in the morning and in the evening on cleansed skin.

Contraindications include: personal hypersensitivity, persons with dark complexion. The preparation should not come in contact with eyes, nose mucous membranes or lips. Azelaic acid should not be used by pregnant and lactating women, due to the lack of precise information on its safety in this respect.

\section{Mandelic acid}

Mandelic acid $\left[\mathrm{HOCH}\left(\mathrm{C}_{6} \mathrm{H}_{5}\right)\right]$ is an organic chemical compound (aromatic hydroxy carboxylic acid), classified as short chain fruit acids - AHA ( $\alpha$-hydroxy acids). It is obtained through hydrolysis of bitter almonds extract.

It displays antibacterial, keratolytic activity and regulates the work of sebaceous glands, and its exfoliating activity regenerates and propagates regeneration of epidermis keratinocytes. With those activities it inhibits the development of micro-comedones. Treatments can be performed at any season of the year on a very sensitive skin (phototype I), and on a very dark skin (phototype VI) without the risk of complications.

Treatments with the use of mandelic acid are characterised by good tolerance. They are successfully used by cosmetologists because of the infinitesimal irritating properties and the fact that they do not sensitize the skin to solar radiation. Mandelic peeling displays the strongest antibacterial activity among all fruit acids, and positively affects the process of proper keratosis of epidermis. Additionally, it levels the skin tone. Chemabrasion treatments based on mandelic acid are good solutions for people suffering from comedonal acne or comedopapular acne [56].

At low concentrations (5-10\%) it is used for delicate care of fatty skin with the tendency to develop comedones. Thanks to the peeling properties, it is also used in anti-wrinkle activity. In aesthetic medicine mandelic acid is used in concentrations from 30 to $70 \%$, however, the most popular are $50 \%, 60 \%$, and $70 \%$ preparations, which are used in acne, and to remove post-solar lesions, pro-inflammatory discolourations, freckles or chloasma. Peelings with mandelic acid should be repeated every 7-15 days, in a series of 6-8 treatments.

Contraindications to mandelic acid peeling include: atopic dry skin prone to irritation, allergy to any ingredients of the preparation used, simultaneous oral treatment with isotretinoin, infectious inflammatory diseases of facial skin, and pregnant and lactating women.

\section{Salicylic acid}

Salicylic acid is an organic compound from the group of aromatic hydroxy carboxylic acids $\left(\mathrm{C}_{7} \mathrm{H}_{6} \mathrm{O}_{3}\right)$. LOcal activity of salicylic acid on skin is based on the reduction of its $\mathrm{pH}$ which improves its protective properties against bacterial, viral, or fungicidal pathogens, causes exfoliation of the superficial layers of epidermis (peeling properties), depending on the concentration, which removes not only the named pathogens from the skin but it also induces faster regeneration of epidermis keratinocytes. 
Salicylic acid is often used for oily skin and both in inflammatory and non-inflammatory acne. Before its use it is always required to check the patient's history of allergy to salicylates. Like other $\alpha$-hydroxy acids, it causes the exfoliation of dead epidermis cells but also due to its fat solubility, it easily permeates the lipid mantle of the skin, thus contributing to the regulation of excessive production of sebum. It is also used in therapies supplementary to folliculitis [57, 58]. In studies carried out on a group of Hindi patients, the effectiveness of $30 \%$ salicylic acid and 45\% mandelic acid was compared. In both cases the condition of facial skin of the patients improved. What is interesting, it was proven that salicylic peeling showed better results in case of non-inflammatory lesions, whereas mandelic peeling showed better results in case of coexisting inflammatory lesions. Mandelic peeling tolerance was much better [59]. Cosmetic preparations with salicylic acid act gently, therefore they are recommended for use in home care for acne skin (it is applied in lower concentrations than in salon treatments).

\section{Glycolic acid}

It is an organic chemical compound classified as $\alpha$-hydroxy acids $\left(\mathrm{C}_{2} \mathrm{H}_{4} \mathrm{O}_{3}\right)$. It is obtained in the reaction of chloroacetic acid and sodium hydroxide. It may also be isolated from natural sources such as sugar cane, sugar beet, pineapple or grapevine. It is applied in skin in different concentrations, on which the intensity of the action mainly depends.

In concentrations up to $15 \%$ glycolic acid displays moisturising activity, causes delicate exfoliation of epidermis and promotes renovation and regeneration of dermis through stimulation of fibroblasts activity.

Concentrations (20-35\%) besides the actions specified above, also peel the skin surface.

High concentrations (50-70\%) display peeling activity and stimulate regenerative processes in dermis (activation of fibroblasts), reconstruction of elastic fibres, which improves skin elasticity. Use of high concentration acids should be done under physician's control.

Treatments with the use of glycolic acid are safe and well-tolerated. Thanks to low molecular weight, this acid permeates well inside the epidermis. It is an effective compound used as a support to acne therapy, especially in mature persons, due to the possibility to improve the turgor of the skin, and in the consequence the reduction of wrinkle visibility [60].

\section{Microdermabrasion}

Another form of procedures to be offered to clients is microdermabrasion - a type of mechanical peeling. It consists in the removal of dead cells as a result of use of a specialist apparatus exfoliating the epidermis with spinning quartz crystals. Microdermabrasion is the most frequent form of mechanical exfoliation used by cosmetologists. The method frequently used by cosmetologists is the performance of chemical peeling after microdermabrasion, or its combination with mesotherapy [3].

The number and frequency of peeling procedures is always agreed upon with the clients because it depends on the kind/type of the skin, the selected method of abrasion and other factors such as the frequency of visits or financial possibilities. To achieve the satisfactory effect it is important to inform the client about the fact that microdermabrasion peeling is usually performed in series, and at intervals specified by the specialist. An important fact is that EDF guidelines from 2016 do not take any stand as to recommendation of the microdermabrasion method nor even mention this method at all.

\section{Light therapies}

There are a lot of controversies regarding the effect of natural sun rays on acneiform lesions, and at this moment there is no significant evidence to prove their positive or negative influence. Sun blocking preparations, however, may cause pro-comedogenic activity and intensify the manifestations of acne.

In UVA/UVB rays tests of blue-red, blue, purple, green light or full spectrum light (FSL) visible or visible and infrared it was demonstrated that acne improved, however, due to some methodology deviations, there are some significant limitations as regards their proper interpretation [61-65].

Ultraviolet (UV) radiation, until recently quite often used to treat acne due to its anti-inflammatory activity, is no longer recommended. It causes the production of squalene peroxide which produced favourable conditions for the formation of new, numerous comedones [65]. The proof is a temporary or even significant improvement of complexion condition during the summer period (the operation of natural UV rays) and very frequent significant deterioration of acne during the autumn period.

LEDs with the use of blue light have been tested quite well in numerous clinical trials. Their positive effect on the inflammatory condition of the skin has been proven in case of mild and moderate form of acne. Moreover, they were well-tolerated and no adverse effects were reported. In the paper of Goldberg et al. there has been noted an exceptional effectiveness of the connection of blue light (415 nm) and red light (633 nm) in acne therapy with different intensity of skin lesions [65-67].

The following methods were used with very different results: photodynamic therapy, pulsed dye laser (PDL), potassium titanyl phosphate (KTP), and intense pulsed light (IPL) [50-52, 67].

Photodynamic therapy (PDT) is based on different monochromatic light sources which activate locally applied phototoxic compounds, e.g. aminolevulinic acid $(A L A)$ or its methyl derivative (MAL) to release free oxygen radicals of biological activity. Among other, they cause destruction of $C$. acnes bacteria existing in sebaceous glands and tubes leading out from them. After the 
treatments, any exposure to sunlight must be avoided due to the possibility of phototoxic reaction of the face skin. It requires individually adjusted exposure time, depending on the radiation energy. It is particularly recommended to persons who cannot be treated with retinoids, with their face, cleavage and back covered with acneiform lesions [52, 54, 68, 69].

An element that distinguishes the IPL therapy is that the apparatus emits radiation with different wave lengths in a very wide spectrum of radiation from 400 to 1600 . The assessment of this method of treatment is very diversified; according to some sources, IPL treatments on acneiform skin are efficient, other claim that it is completely ineffective [65].

In supplementary therapy of acne vulgaris there are also used treatments with more or less invasive lasers (Er:YAG, CO2, fraction, diode, mosaic type lasers) performed by specialist doctors. Laser treatments of this type are mainly used to reduce post-acne scars, especially those of atrophic nature. They may considerably improve the looks of the skin, even after undergoing very intense forms of acne vulgaris but they must be performed by doctors with an extensive experience in those types of therapies [65, 70].

\section{Combined methods}

Combination of various local therapy methods brings the largest benefits in the treatment of scars and discolourations resulting from the most severe forms of acne. Sometimes, in order to obtain the best results, a close cooperation between a cosmetologist, dermatologist and plastic surgeon is necessary. The most frequently used combined methods include:

- Local retinoids, vitamin C, E preparations, hydroquinone derivatives with subsequent microdermabrasion treatments.

- Microdermabrasion with subsequent chemical peelings ( $\alpha$-hydroxy acids, TCA, Jessner's solution, etc.).

- Mechanical, laser abrasion treatments with subsequent use of external immunomodulators (pimecrolimus, tacrolimus).

- Laser thermabrasion treatments (Er:YAG, $\mathrm{CO}_{2}$ ) with cryosurgery treatments (cryopeeling, contact method).

- Chemical peelings with non-ablative laser techniques or intense pulsed lights (IPL).

- Cryosurgery methods with intralesional corticosteroid injections.

- Plastic surgery corrections with subsequent application of aesthetic dermatology methods (dermabrasions chemical, mechanical, laser, etc.).

\section{Summary}

Acne vulgaris is one of the most common dermatosis among young population come to dermatological and cosmetology consultation. The disease has a significant, negative effect on the quality of life of young people, especially girls, who in the majority of cases (71\%) do not accept their appearance [71].

Before commencing any skincare or medicinal treatment, one must get a complete anamnesis, and information on nutritional habits, aggravation factors, the course and treatment up to date, recognise the clinical form of acne and specify the severity level, and note the mental condition of the patient, which seems to be very important when choosing the right proceedings.

Proper care combined with local therapy is sufficient in over $60 \%$ of patients with excessive facial skin seborrhoea, or mild comedonal acne and comedo-papular acne.

It must be remembered that in cosmetologist's practice it is advisable to combine different treatment forms. The combination of e.g. abrasive therapies (mechanical, chemical or physical) may unclog the retention of sebum in the glands and improve the effects of dermatological treatment.

Skincare treatments, including the abrasive ones, should be adjusted individually to each patient, considering:

- the season of a year,

- age and sex,

- diet,

- psychological profile,

- severity of seborrhoea,

- skin sensitivity level,

- skin phototype,

- the presence of telangiectasia or discolourations.

Local therapy of acne and cosmetology and dermatosurgery procedures significantly improve the appearance and limit the occurrence of acneiform lesions and post-acne complications (scars), and in the consequence lead to the improvement of the quality of life of young patients. Therefore, the appropriate cosmetology procedure should be undertaken in cooperation with a dermatologist [21].

\section{Conflict of interest}

The authors declare no conflict of interest.

\section{References}

1. Barbaric J, Abbott R, Posadzki P, et al. Light therapies for acne. Cochrane Database Syst Rev 2016; 9: CD007917.

2. Vilar GN, Santos LA, Sobral Filho JF. Quality of life, self-esteem and psychosocial factors in adolescents with acne vulgaris. An Bras Dermatol 2015; 90: 622-9.

3. Adamski Z, Kaszuba A. Choroby tojotokowe skóry. In: Dermatologia dla kosmetologów. Adamski Z, Kaszuba A (eds.). Elsevier Urban Partner, Wrocław 2010; 54-6, 290-3, 379-80.

4. Costa CS, Bagatin E, Martimbianco ALC, et al. Oral isotretinoin for acne. Cochrane Database Syst Rev 2018; 11: CD009435. 
5. Szepietowski J, Kapińska-Mrowiecka M, Kaszuba A, et al. Trądzik zwyczajny: patogeneza i leczenie. Konsensus Polskiego Towarzystwa Dermatologicznego. Przegl Dermato 2012; 99: 649-73.

6. Poli F, Dreno B, Verschoore M. An epidemiological study of acne in female adults: results of a survey conducted in France. J Eur Acad Derm Venerol 2001; 15: 541-5.

7. George R, Clarke S, Thiboutot D. Hormonal therapy for acne. Semin Cutan Med Surg 2008; 27: 188-96.

8. Cunliffe WJ, Gollnick HPM. Acne. Diagnosis and management. Martin Dunitz, London 2001.

9. Gollnick H. Current concepts of the pathogenesis of acne: implications for drug treatment. Drugs 2003; 63: 1579-96.

10. Jeremy AH, Holland DB, Roberts SG, et al. Inflammatory events are involved in acne lesion initiation. J Invest Dermatol 2003; 121: 20-7.

11. Makrantonaki E, Ganceviciene R, Zouboulis C. An update on the role of the sebaceous gland in the pathogenesis of acne. Dermatoendocrinology 2011; 3: 41-9.

12. Zhang HY, Rong FF, Feng WL, et al. 3 cases with occupational chloracne caused by sodiumtrichloropyridine. Zhonghua Lao Dong Wei Sheng Zhi Ye Bing Za Zhi 2019; 20: 301-2.

13. Chiu A, Chon SY, Kimball AB. The response of skin disease to stress: changes in the severity of acne vulgaris as affected by examination stress. Arch Dermatol 2003; 139: 897-900.

14. Kowalska H, Sysa-Jędrzejowska A, Woźniacka A. Rola diety w etiopatogenezie trądziku. Przegl Dermatol 2018; 105: 51-62.

15. Melnik BC, John SM, Schmitz G. Over-stimulation of insulin/ IGF-1 signaling by western diet may promote diseases of civilization: lessons learnt from Laron syndrome. Nutr Metab 2011; 8: 41.

16. Adebamowo CA, Spiegelman D, Danby FW, et al. High school dietary dairy intake and teenage acne. I Am Acad Dermato 2005; 52: 207-14.

17. Wolkenstein P, Machovcová A, Szepietowski JC, et al. Acne prevalence and associations with lifestyle: a crosssectional online survey of adolescents/young adults in 7 European countries. J Eur Acad Dermatol Venereol 2018; 32: 298-306

18. Rombouts S, Nijsten T, Lambert J. Cigarette smoking and acne in adolescents: results from a cross-sectional study. J Eur Acad Derm Venerol 2007; 21: 326-33.

19. Hauk L. Acne vulgaris: treatment guidelines from the AAD. Am Fam Physician 2017; 95: 740-1.

20. Das S, Reynolds RV. Recent advances in acne pathogenesis: implications for therapy. Am J Clin Dermatol 2014; 15: 479-88.

21. Chilicka K, Maj J, Panaszek B. General quality of life of patients with acne vulgaris before and after performing selected cosmetological treatments. Patient Prefer Adherence 2017; 11: 1357-61.

22. Marson JW, Baldwin HE. An overview of acne therapy, part 1: topical therapy, oral antibiotics, laser and light therapy, and dietary interventions. Dermatol Clin 2019; 37: 183-93.

23. Szepietowski J, Kapińska-Mrowiecka M, Kaszuba A, et al. Stanowisko grupy eksperckiej Polskiego Towarzystwa Dermatologicznego w sprawie konsensusu „Trądzik zwyczajny: patogeneza i leczenie". Przegl Dermatol 2014; 101: 455-72.

24. Gollnick H, Cunliffe W, Berson D, et al. Management of acne: a report from a global alliance to improve outcomes in acne. J Am Acad Dermatol 2003; 49: S1-37.

25. Krakowski AC, Stendardo S, Eichenfield LF. Practical considerations in acne treatment and the clinical impact of topical combination therapy. Pediatr Dermatol 2008; 25 (Suppl 1): $1-14$
26. Worret WI, Fluhr JW. Acne therapy with topical ben-zoyl peroxide, antibiotics and azelaic acid. J Dtsch Dermatol Ges 2006; 4: 293-300

27. Nast A, Dréno B, Bettoli V, et al. European evidence-based (S3) guidelines for the treatment of acne. J Eur Acad Derm Venerol 2012; 26 (supl. 1): 1-29.

28. Zaenglein AL, Thiboutot DM. Expert Committee recommendations for acne management. Pediatrics 2006; 118: 11882000.

29. Leyden J, Preston N, Osborn C, et al. In-vivo effectiveness of adapalene $0.1 \%$ benzoyl peroxide $2.5 \%$ gel on antibiotic sensitive and resistant Propionibacterium acnes. J Clin Aesthet Dermatol 2011; 4: 22-6.

30. Kose O, Koc E, Arca E. Adapalene gel $0.1 \%$ in the treatment of infantile acne: an open clinical study. Pediatr Dermatol 2008; 25: 383-6.

31. Geng A, Weinstock MA, Hall R, et al. Tolerability of high-dose topical tretinoin: the veterans affairs topical tretinoin chemoprevention trial. Br J Dermatol 2009; 161: 918-24.

32. Thielitz A, Krautheim A, Gollnick H. Update in retinoid therapy of acne. Dermatol Ther 2006; 19: 272-9.

33. Lucky AW, Sugarman J. Comparison of micronized tretinoin gel $0.05 \%$ and tretinoin gel microsphere $0.1 \%$ in young adolescents with acne: a post hoc analysis of efficacy and tolerability data. Cutis 2011; 87: 305-10.

34. Langner A, Sheehan-Dare R, Layton A. A randomized, singleblind comparison of topical clindamycin+benzoyl peroxide $\left(\right.$ Duac $\left.^{\circledast}\right)$ and erythromycin + zinc acetate $\left(\right.$ Zineryt $\left.^{\circledR}\right)$ in the treatment of mild to moderate facial acne vulgaris. J Eur Acad Derm Venerol 2007; 21: 311-9.

35. Leyden JJ. A review of the use of combination therapies for the treatment of acne vulgaris. J Am Acad Dermatol 2003; 49 (supl. 3): S200-10.

36. Gollnick HP, Draelos Z, Glenn MJ, et al. Adapalene-benzoyl peroxide, a unique fixed dose combination topical gel for the treatment of acne vulgaris: a transatlantic, randomized, double-blind, controlled study in 1670 patients. Br J Dermatol 2009; 161: 1180-9.

37. Thiboutot D, Gollnick H, Bettoli V, et al. New insights into the management of acne: an update from the Global Alliance to Improve Outcomes in Acne Group. I Am Acad Dermatol 2009; 60 (Supl. 5): S1-50.

38. Shalita A. The integral role of topical and oral retinoids in the early treatment of acne. J Eur Acad Derm Venerol 2001; 15 (Supl. 3): 43-9.

39. Gelmetti C. Local antibiotics in dermatology. Dermatol Ther 2008; 21: 187-95.

40. Simonart T, Dramaix M. Treatment of acne with topical antibiotics: lesson from clinical studies. Br J Dermatol 2005; 153: 395-403.

41. Ross JI, Snelling AM, Carnegie E, et al. Antibiotic-resistant acne: lessons from Europe. Br J Dermatol 2003; 148: 467-78.

42. Katsambas AD. Why and when the treatment of acne fails. What to do? Dermatology 1998; 196: 158-61.

43. Dreno B. Topical antibacterial therapy for acne vulgaris. Drugs 2004; 64: 2389-97.

44. Ozolins M, Eady EA, Avery AJ, et al. Comparison of five antimicrobial regiment for treatment of mild to moderate inflammatory facial acne vulgaris in the community: randomized controlled trial. Lancet 2004; 364: 2188-95.

45. Tucker SB, Tausend R, Cochran R, et al. Comparison of topical clindamycin phosphate, benzoyl peroxide, and a combination of the two for the treatment of acne vulgaris. Br J Dermatol 1984; 110: 487-92. 
46. Charakida A, Charakida M, Chu AC. Double-blind, randomized, placebo-controlled study of a lotion containing triethyl citrate and ethyl linoleate in the treatment of acne vulgaris. Br J Dermatol 2007; 157: 569-74.

47. Burkhart CG, Burkhart CN, Isham N. Expanded yeast and bacterial coverage: synergistic activity by free radicals fordem by combining an allylamine with benzoyl peroxide. Br J Dermatol 2006; 154: 341-4.

48. Leyden J, Thiboutot DM, Shalita AR, et al. Comparison of tazarotene and minocycline maintenance therapies in acne vulgaris: a multicenter, double-blind, randomized, parallelgroup study. Arch Dermatol 2006; 142: 605-12.

49. Kapińska-Mrowiecka M. Postępowanie pielęgnacyjne w trądziku. Dermatol i Uroda, Jesień-Zima 2011.

50. Nouri K, Ballard CJ. Laser therapy for acne. Clin Dermatol 2006; 24: 26-32.

51. Leheta TM. Role of the 585-nm pulsed dye laser in the treatment of acne in comparison with other topical therapeutic modalities. J Cosmet Laser Ther 2009; 11: 118-24.

52. Hongcharu W, Taylor CR, Chang Y. Topical ALA-photodynamic therapy for the treatment of acne vulgaris. J Invest Dermatol 2000; 115: 183-92.

53. Choi YS, Suh HS, Yoon MY. Intense pulsed light vs. pulseddye laser in the treatment of facial acne: a randomized splitface trial. J Eur Acad Derm Venerol 2010; 24: 773-80.

54. Tong LX, Brauer JA. Lasers, light, and the treatment of acne: a comprehensive review of the literature. J Drugs Dermatol 2017; 16: 1095-102.

55. Szymańska A, Budzisz E, Ekiert-Polguj A. Efficacy of 30\% azelaic acid peel in the nonpharmacological treatment of facial acne. J Dermatolog Treat 2019; Aug 28: 1-6. doi: 10.1080/09546634.2019.1657222.

56. Sarkar R, Ghunawat S, Garg VK. Comparative study of 35\% glycolic acid, $20 \%$ salicylic-10\% mandelic acid, and phytic acid combination peels in the treatment of active acne and postacne pigmentation. J Cutan Aesthet Surg 2019; 12: 158-63.

57. Lekakh O, Mahoney AM, Novice K, et al. Treatment of acne vulgaris with salicylic acid chemical peel and pulsed dye laser: a split face, rater-blinded, randomized controlled trial. J Lasers Med Sci 2015; 6: 167-70.

58. Kligman DE, Draelos ZD. Combination superficial peels with salicylic acid and post-peel retinoids. J Drugs Dermatol 2016; 15: 442-50.

59. Dayal S, Kalra KD, Sahu P. Comparative study of efficacy and safety of $45 \%$ mandelic acid versus $30 \%$ salicylic acid peels in mild-to-moderate acne vulgaris. J Cosmet Dermatol 2020; 19: 393-9.

60. Al-Talib H, Al-Khateeb A, Hameed A, et al. Efficacy and safety of superficial chemical peeling in treatment of active acne vulgaris. An Bras Dermatol 2017; 92: 212-6.

61. Mills $\mathrm{OH}$, Kligman AM. Ultraviolet phototherapy and photochemotherapy of acne vulgaris. Arch Dermatol 1978; 114: 221-3.

62. Ammad S, Gonzales M, Edwards C, et al. An assessment of the efficacy of blue light phototherapy in the treatment of acne vulgaris. J Cosmet Dermatol 2008; 7: 180-8.

63. Kawada A, Aragane Y, Kameyama H, et al. Acne phototherapy with a high-intensity, enhanced, narrow-band, blue light source: an open study and in vitro investigation. J Dermatol Sci 2002; 30: 129-35.

64. Sigurdsson V, Knulst AC, van Weelden H. Phototherapy of acne vulgaris with visible light. Dermatology 1997; 194: 25660.
65. Degitz K. Phototherapy, photodynamic therapy and lasers in the treatment of acne. J Dtsch Dermatol Ges 2009; 7: 1048-54. 66. Goldberg DJ, Russell BA. Combination blue $(415 \mathrm{~nm})$ and red $(633 \mathrm{~nm})$ LED phototherapy in the treatment of mild to severe acne vulgaris. J Cosmet Laser Ther 2006; 8: 71-5.

67. Sadick N. A study to determine the effect of combination blue $(415 \mathrm{~nm})$ and near-infrared $(830 \mathrm{~nm})$ light-emitting diode (LED) therapy for moderate acne vulgaris. J Cosmet Laser Ther 2009; 11: 125-8.

68. Elman M, Lebzelter J. Light therapy in the treatment of acne vulgaris. Dermatol. Surg 2004; 30: 139-46.

69. Bissonnette R. Treatment of acne with photodynamic therapy. G Ital Dermatol Venereol 2011; 146: 445-56.

70. Friedman PM, Jih MH, Kimyai-Asadi A, et al. Treatment of inflammatory facial acne vulgaris with the $1450 \mathrm{~nm}$ diode laser: a pilot study. Dermatol Surg 2004; 30: 147-51.

71. Sobkowska D, Męczkalski B, Gornowicz-Porowska J, et al. Ocena jakości życia u kobiet z nasilonymi zmianami trądzikowymi z regionu wielkopolski. Derm Prakt 2019; 2: 19-27. 